\title{
A high-sensitivity differential scanning calorimeter with magnetic field for magnetostructural transitions
}

\author{
Jordi Marcos, Fèlix Casanova, Xavier Batlle, Amílcar Labarta, Antoni Planes, \\ and Lluís Mañosa ${ }^{\text {a) }}$ \\ Facultat de Física, Universitat de Barcelona, Diagonal, 647, E-08028 Barcelona, Catalonia, Spain
}

(Received 17 January 2003; accepted 18 July 2003)

\begin{abstract}
We have developed a differential scanning calorimeter capable of working under applied magnetic fields of up to $5 \mathrm{~T}$. The calorimeter is highly sensitive and operates over the temperature range $10-300 \mathrm{~K}$. It is shown that, after a proper calibration, the system enables determination of the latent heat and entropy changes in first-order solid-solid phase transitions. The system is particularly useful for investigating materials that exhibit the giant magnetocaloric effect arising from a magnetostructural phase transition. Data for $\mathrm{Gd}_{5}\left(\mathrm{Si}_{0.1} \mathrm{Ge}_{0.9}\right)_{4}$ are presented. (c) 2003 American Institute of Physics. [DOI: 10.1063/1.1614857]
\end{abstract}

\section{INTRODUCTION}

Calorimetry has been used to study a number of physical properties of solids for more than a century. ${ }^{1}$ Even now, it is considered to be a well suited method to determine the nature of phase transitions. Currently, a wide variety of calorimeters exist, which can be broadly classed into two groups. The first group includes those devices which measure the heat flux between the sample and a thermal block, while the temperature of the calorimeter is continuously changed (scanning calorimeters). Most of them use a dummy sample so that they work differentially [differential scanning calorimeters (DSCs)]. The second group includes the calorimeters which are based on the measurement of the temperature of the sample after a small amount of heat is supplied (adiabatic calorimetry, relaxation calorimetry, and ac calorimetry). In these instruments, the temperature of the calorimeter is kept constant during the measurement. There are also calorimeters which combine the two operating methods, as for instance in the case of the modulated differential scanning calorimeters which have been recently developed. ${ }^{2}$ Continuous efforts are devoted to designing calorimeters that are better adapted to the new materials and with better performances. ${ }^{3,4}$

DSCs are particularly suited to studying first-order phase transitions since they measure the heat flux, and a proper integration of the calibrated signal yields the latent heat of the transition. In contrast, ac, relaxation, and adiabatic calorimetry are suitable for determining the specific heat and therefore, are well adapted for studying continuous phase transitions. It should be noted that in a first-order transition, a heat input does not result in a modification of the temperature of the sample and, therefore, the latter techniques are not suitable for studying this kind of phase transition.

A number of devices have been developed which are aimed at studying the effect of the magnetic field on the thermal properties of phase transitions. Among them, particularly interesting are those which measure adiabatic tem-

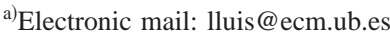

perature changes associated with the application or removal of a magnetic field (magnetocaloric effect), ${ }^{5,6}$ and a highsensitivity differential thermal analysis (DTA) device which measures the specific heat at the vortex lattice melt of high $T_{c}$ superconductors. $^{7}$ Nevertheless, in many materials the transition is first order, with characteristics extremely sensitive to the application of a magnetic field. In these cases, a DSC is the ideal tool to obtain the latent heat and entropy changes associated with these transitions since DTA can just provide an upper limit for the latent heat. In the present article we describe a high-sensitivity differential scanning calorimeter which operates over a temperature range from 10 to $300 \mathrm{~K}$ and under magnetic fields of up to $5 \mathrm{~T}$. This calorimeter provides accurate values of the latent heat and entropy change at a first-order phase transition under magnetic field. As a case study, it is used to obtain the field dependence of the latent heat and entropy change in $\mathrm{Gd}_{5} \mathrm{Si}_{0.4} \mathrm{Ge}_{3.6}$, which displays the giant magnetocaloric effect. ${ }^{8}$

\section{EXPERIMENTAL DETAILS}

Figure 1 shows (a) side and (b) top cross sections of the calorimeter. The calorimeter can be adapted to any cryostat equipped with a superconducting magnet. The apparatus described here has the appropriate size to be used with a Teslatron $($ (Oxford Instruments) system as a host platform. A magnetic field of up to $5 \mathrm{~T}$ is generated along the vertical axis by a superconducting magnet included in the Teslatron system. The calorimeter is a copper spool (1). ${ }^{9}$ It is mechanically clamped to a long stainless steel tube. All wiring is routed through this rod and exits the system via an electrical feedthrough at its far end. The two sensors (2), which are differentially connected, are placed on the flattened inner surfaces of the spool. In order to ensure a good thermal contact, the sensors are coupled to the block with General Electrics Oxford Varnish. These sensors are batteries of thermocouples (Melcor FCO.45-32-05L) ${ }^{10}$ made of $P-N$ junctions of $\mathrm{Bi}_{2} \mathrm{Te}_{3}$ (32 pairs of junctions on a $6.5 \times 6.5 \mathrm{~mm}^{2}$ surface). Sample (3) and inert reference (4) are placed directly on top of each sensor. They are held in place (in good thermal contact with 


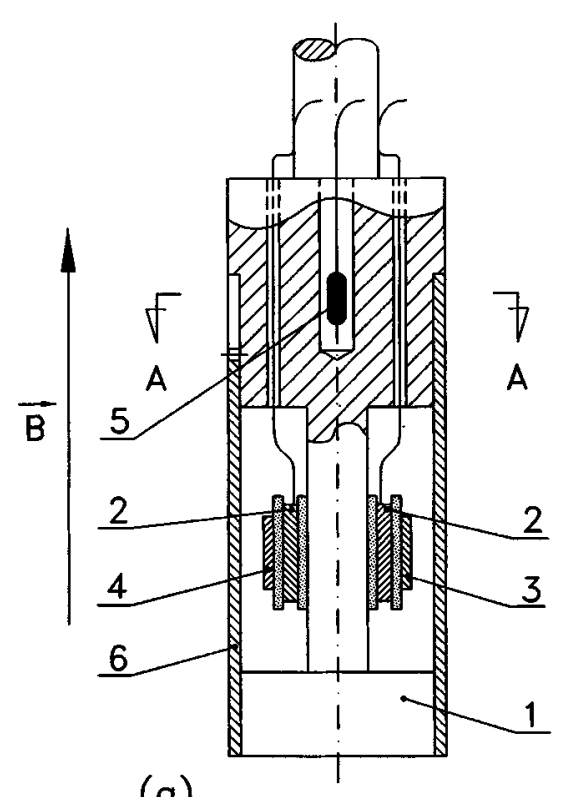

(a)
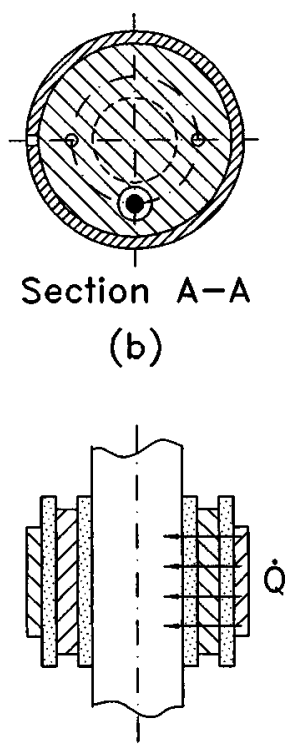

(c)

FIG. 1. (a) Side and (b) top view cross sections of the calorimeter. (1) Copper spool, (2) sensors, (3) sample, (4) reference, (5) carbon-glass resistor, and (6) cover. The magnetic field, $B$, is along the symmetry axis of the calorimeter; (c) detail showing the heat flow $\dot{Q}$ for an exothermal transition.

the sensor) by winding a thin (less than $0.2 \mathrm{~mm}$ diameter) nylon wire around the assembly. Electrical wires exit the calorimeter through $2.5 \mathrm{~mm}$ diameter holes, and they are thermally coupled to the upper part of the spool before passing through the stainless steel rod. Such a coupling avoids the existence of thermal gradients on the wires which could give rise to spurious thermoelectric voltages. The temperature of the calorimeter is scanned by changing the temperature of the variable temperature insert (VTI) of the Teslatron cryostat. An accurate reading of the actual temperature of the calorimeter is achieved by monitoring the electrical resistance of a carbon-glass resistor (LakeShore Cryotronics Inc. CGR-1-500) (5) embedded inside the spool. In order to minimize convection of the exchange gas inside the calorimeter, the whole assembly is covered by an external copper cylinder (1 mm thick) (6), which is screwed to the upper part of the spool. For an optimal operation, the pressure inside the calorimeter should be within the range 200-600 mbar. High purity helium is required since impurities affect the sensitivity of the calorimeter.

The heat released (or absorbed) by the sample is measured by reading the voltage furnished by the thermobatteries (electrical output) by using a nanovoltmeter (Keithley 182). It is worth reminding that, since the two sensors are connected differentially, the drift in the calorimetric output associated with changes in the temperature of the calorimetric block are minimized by the fact that the heat flow of the reference is subtracted from that of the sample, and therefore, the major contribution to the calorimetric output is the thermal power released (or absorbed) by the sample during the first-order phase transition (latent heat). The resistance of the carbon glass is read by means of a lockin amplifier (EG \& G 7260). ${ }^{11}$ The whole system (including the electronics of the Teslatron measurement system) was controlled by a PC

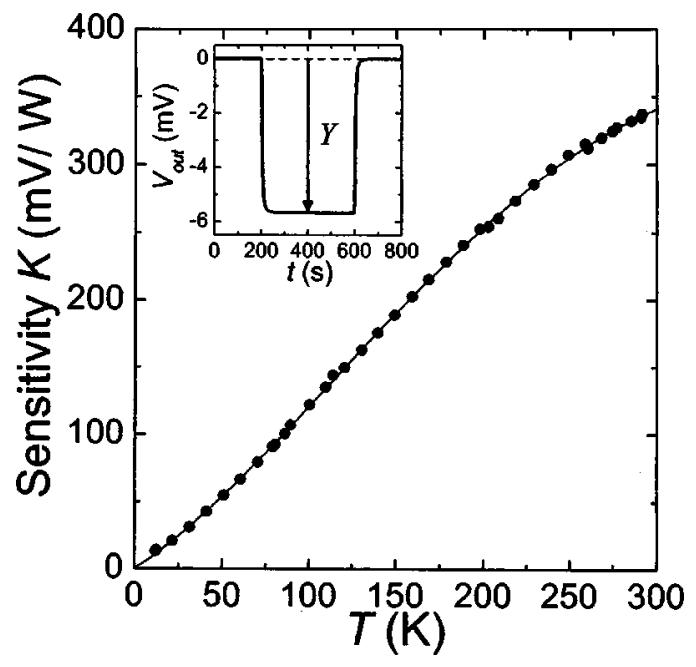

FIG. 2. Sensitivity at zero field as a function of temperature. The solid line is a fit to the data. Inset: example of a typical calibration thermogram at $T$ $=173 \mathrm{~K}$.

computer. Values of voltage and temperature are acquired at typical rates of $0.25 \mathrm{~Hz}$.

Since the thermocouples are made of semiconducting elements, the magnetic field is not expected to affect their thermoelectric output. In contrast, the output voltage will indeed significantly depend on temperature and therefore, a proper calibration over the whole operating temperature range is needed. To carry out such a calibration, the sample is replaced by a manganin resistance $(50 \Omega)$. A constant power $(W)$ is dissipated by the Joule effect at the resistance (without the applied magnetic field), and the electrical output at the steady state, $Y$, is measured. The sensitivity, $K$, is then given by: $K=Y / W$. A typical calorimetric curve for $W=18 \mathrm{~mW}$ is shown in the inset of Fig. 2. ${ }^{12}$ The values obtained for the sensitivity at different temperatures are plotted in Fig. 2. Data can be fitted by the curve: $K(\mathrm{mV} / \mathrm{W})=1.4$ $\times 10^{-8} \mathrm{~T}^{4}-2.0 \times 10^{-5} \mathrm{~T}^{3}+5.1 \times 10^{-3} \mathrm{~T}^{2}+0.86 \mathrm{~T}$, which is also plotted in the figure. It is interesting to note that the room temperature value is around 10 times larger than the sensitivity for a conventional DSC, ${ }^{13}$ and that at temperatures as low as $10 \mathrm{~K}$, a reasonably high value is still obtained.

\section{RESULTS}

To check the performances of the calorimeter, we have selected a $\mathrm{Cu}-\mathrm{Zn}-\mathrm{Al}$ alloy undergoing a structural (martensitic) transition, as a standard system. This material is diamagnetic and hence the transition is not affected by a magnetic field; this will provide a good test of the insensitivity of the sensors to a magnetic field. On the other hand, the values for the latent heat and entropy change associated with the martensitic transition are very well established over a broad temperature range by the use of several experimental techniques (the transition temperature can be modified by slightly tuning the composition). ${ }^{14,15}$ The calorimetric curves recorded during the reverse transition of a $\mathrm{Cu}-\mathrm{Zn}-\mathrm{Al}$ crystal are shown in Fig. 3, in the absence of a magnetic field (continuous line) and for an applied field of $5 \mathrm{~T}$ (discontinuous 


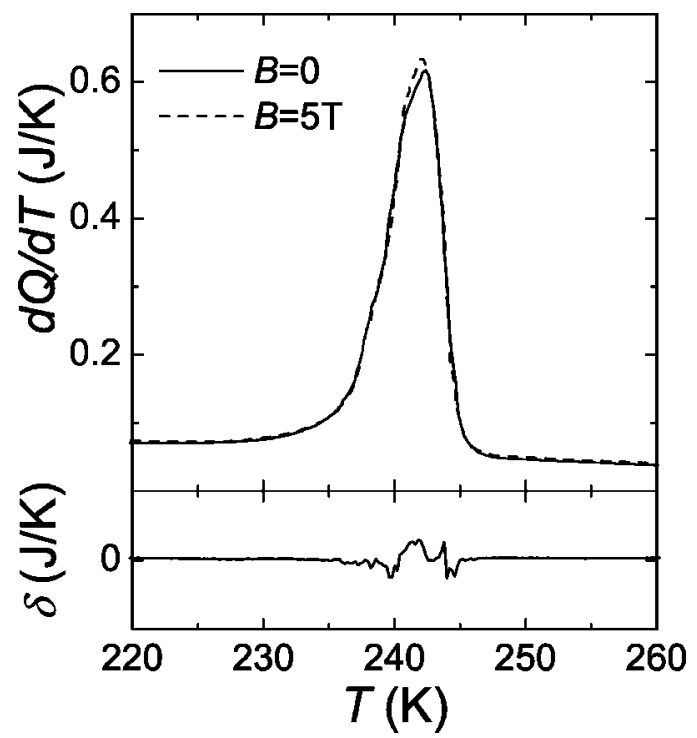

FIG. 3. Calorimetric curves recorded at the martensitic transition of a $\mathrm{Cu}_{2.717} \mathrm{Zn}_{0.646} \mathrm{Al}_{0.637}$ single crystal at zero field (continuous curve) and at $B$ $=5 \mathrm{~T}$ (discontinuous curve). The difference between these two curves $\delta$ is also shown.

curve). Since the two curves are almost indistinguishable, we have also plotted the difference between them, $\delta$. No significant influence of the magnetic field is observed.

In order to obtain the latent heat and the entropy change in a first-order phase transition, the calorimetric signal has to be corrected from the baseline (details can be found, for instance, in Ref. 16). The latent heat and the entropy change are then given by

$$
\Delta H=\int_{T_{L}}^{T_{H}} \frac{d Q}{d T} d T ; \quad \Delta S=\int_{T_{L}}^{T_{H}} \frac{1}{T} \frac{d Q}{d T} d T,
$$

where $T_{H}$ and $T_{L}$ are, respectively, temperatures above and below the starting and finishing transition temperatures. The values obtained for the latent heat $[\Delta H(B=0)=336$ $\pm 3 \mathrm{~J} / \mathrm{mol}$, and $\Delta H(B=5 \mathrm{~T})=335 \pm 3 \mathrm{~J} / \mathrm{mol}]$ and for the entropy change $[\Delta S(B=0)=1.40 \pm 0.01 \mathrm{~J} / \mathrm{mol} \mathrm{K}$ and $\Delta S(B$ $=5 \mathrm{~T})=1.39 \pm 0.01 \mathrm{~J} / \mathrm{mol} \mathrm{K}]$ at the martensitic transition of $\mathrm{Cu}-\mathrm{Zn}-\mathrm{Al}$ are in excellent agreement with published values. ${ }^{14,15}$

The apparatus described in this article is particularly well adapted for measuring the entropy change at the magnetostructural phase transition undergone by alloys which exhibit the giant magnetocaloric effect. The determination of the entropy change associated with the magnetocaloric effect in these materials has been an issue of controversial debate. ${ }^{8,17-20}$ Proper measurement of the entropy change associated with the phase transition is expected to contribute to a better understanding of this interesting phenomenon. Figure 4 shows an example of the thermal curves recorded during heating and cooling of a $\mathrm{Gd}_{5}\left(\mathrm{Si}_{0.1} \mathrm{Ge}_{0.9}\right)_{4}$ sample under different applied magnetic fields. The small peak at around $130 \mathrm{~K}$ arises from a second-order paramagnetic to antiferromagnetic phase transition. ${ }^{8}$ The large peak at lower temperatures is due to a first-order phase transition between two different orthorhombic structures. An antiferromagneticferromagnetic transition occurs simultaneously with this

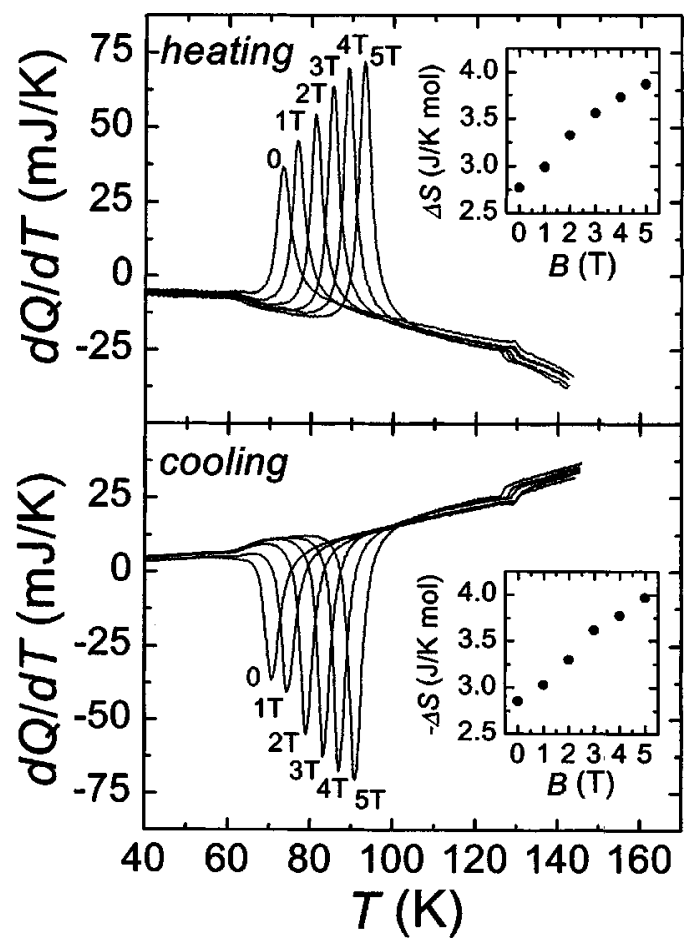

FIG. 4. Calorimetric curves recorded in the forward and reverse transitions of a $\mathrm{Gd}_{5}\left(\mathrm{Si}_{0.1} \mathrm{Ge}_{0.9}\right)_{4}$ sample for different applied magnetic fields. Insets show the entropy change as a function of magnetic field.

structural transition. ${ }^{21}$ The thermal hysteresis amounts to $2-3$ $\mathrm{K}$. The magnetic field dependence of the first-order phase transition is evident from the calorimetric curves. A linear increase with the magnetic field is obtained for the transition temperature, with a slope $(=4.1 \pm 0.1 \mathrm{~K} / \mathrm{T})$ which is in agreement with that derived from magnetization measurements. ${ }^{21}$ The entropy change at the transition has also been found to increase with magnetic field, as shown in the insets of Fig. 4. For $B=0, \Delta S=2.85 \mathrm{~J} / \mathrm{mol} \mathrm{K}$ while for $B=5 \mathrm{~T}, \Delta S$ $=3.96 \mathrm{~J} / \mathrm{mol} \mathrm{K}$. Such an increase is a consequence of the coupling between structural and magnetic degrees of freedom. It is worth remarking that the entropy values derived from isothermal magnetization curves using the ClausiusClapeyron equation coincide with the values measured calorimetrically. A thorough discussion of the measurements on a series of samples will be given elsewhere. ${ }^{22}$

To conclude, a differential scanning calorimeter has been developed which features a high sensitivity down to $10 \mathrm{~K}$, and which operates under applied magnetic fields up to $5 \mathrm{~T}$. The device has been used to study first-order solid-solid phase transitions in the presence of magnetic fields. It has been shown that it enables an accurate determination of the entropy change in the magnetostructural phase transition of alloys exhibiting the giant magnetocaloric effect.

\section{ACKNOWLEDGMENTS}

This work has received financial support from the CICyT (Spain), Project Nos. MAT2000-0858 and MAT20013251, and from the CIRIT (Catalonia), Project No. 2001SGR00066. J.M. and F.C. acknowledge DURSI for Ph.D. Grants. 
${ }^{1}$ For a recent review on calorimetry see, Y. Kraftmakher, Phys. Rep. 356, 1 (2002).

${ }^{2}$ O. S. Gill, S. R. Suerbrunn, and M. Reading, J. Therm. Anal. 40, 931 (1993).

${ }^{3}$ I. K. Moon, D. H. Jung, K.-B. Lee, and Y. H. Jeong, Appl. Phys. Lett. 76, 2451 (2000).

${ }^{4}$ A. I. Kharkovski, Ch. Binek, and W. Kleemann, Appl. Phys. Lett. 77, 2409 (2000).

${ }^{5}$ B. R. Gopal, R. Chahine, and T. K. Bose, Rev. Sci. Instrum. 68, 1818 (1997).

${ }^{6}$ S. Yu. Dan'kov, A. M. Tishin, V. K. Pecharsky, and K. A. Gschneidner, Jr., Rev. Sci. Instrum. 68, 2432 (1997).

${ }^{7}$ A. Schilling and O. Jeandupeux, Phys. Rev. B 52, 9714 (1995).

${ }^{8}$ V. K. Pecharsky and K. A. Gschneidner, Jr., Appl. Phys. Lett. 70, 3299 (1997).

${ }^{9}$ For optimal performance, the mass of the spool has to be large enough so that its specific heat amounts to at least 100 times that of the sample (or reference).

${ }^{10}$ CMelcor Thermoelectrics, 1040 Spruce St., Trenton, NJ 08648.
${ }^{11} \mathrm{~A}$ microvoltmeter can also be used to read the resistance of the thermometer.

${ }^{12}$ The linearity of the calorimeter has been verified by performing calibrations with different values of the released power.

${ }^{13}$ S. M. Sarge, E. Gmelin, G. W. H. Höhne, H. K. Cammenga, W. Hemminger, and W. Eysel, Thermochim. Acta 247, 129 (1994).

${ }^{14}$ R. Romero and J. L. Pelegrina, Phys. Rev. B 50, 9046 (1994).

${ }^{15}$ A. Planes and L. Mañosa, Solid State Phys. 55, 159 (2001).

${ }^{16}$ J. Ortín, Thermochim. Acta 121, 397 (1987).

${ }^{17}$ A. Giguère, M. Földeàki, B. Ravi Gopal, R. Chahine, T. K. Bose, A. Frydman, and J. A. Barclay, Phys. Rev. Lett. 84, 4617 (2000).

${ }^{18}$ K. A. Gschneider, Jr., V. K. Pecharsky, E. Brück, H. G. M. Duijn, and E. Levin, Phys. Rev. Lett. 85, 4190 (2000).

${ }^{19}$ J. R. Sun, F. X. Hu, and B. G. Shen, Phys. Rev. Lett. 85, 4191 (2000).

${ }^{20}$ M. Földeàki, R. Chahine, T. K. Bose, and J. A. Barclay, Phys. Rev. Lett. 85, 4192 (2000).

${ }^{21}$ L. Morellon, J. Blasco, P. A. Algarabel, and M. R. Ibarra, Phys. Rev. B 62, $1022(2000)$

${ }^{22}$ F. Casanova, X. Batlle, A. Labarta, J. Marcos, L. Mañosa, and A. Planes, Phys. Rev. B 66, 224413 (2002); 66, 212402 (2002). 